\title{
ADVANCES IN ENDOSCOPY
}

\author{
Current Developments in Diagnostic and Therapeutic Endoscopy
}

Section Editor: Todd H. Baron, MD

\section{Endoscopic Ultrasound-Guided Liver Biopsy}

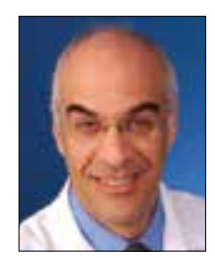

\author{
David L. Diehl, MD \\ Clinical Professor of Medicine and Pathology \\ Director of Interventional Endoscopy \\ Geisinger Commonwealth School of Medicine \\ Department of Gastroenterology and Nutrition \\ Geisinger Medical Center \\ Danville, Pennsylvania
}

\section{G\&H What are the indications for a liver biopsy?}

DD The most common indications for parenchymal liver biopsy are for an assessment of acute or chronic liver disease, an evaluation of the degree of liver fibrosis, and a workup of abnormal liver function tests. Less common indications include exclusion of autoimmune hepatitis, assessment of iron or copper in the liver, or assessment of post-liver transplant patients.

\section{G\&H What approaches are currently used to sample liver tissue?}

DD There are several approaches available to acquire a liver biopsy. The traditional method is a percutaneous biopsy, in which a site is localized on the right side over the liver, a small nick is made in the skin, and a large-gauge needle is plunged into the liver to obtain a biopsy. This method is often performed by interventional radiologists under computed tomography or ultrasound guidance.

Liver tissue can also be sampled via a transjugular route, wherein an interventional radiologist enters through the jugular vein, drops a needle sheath down to the hepatic veins, and samples the liver. A transjugular biopsy is typically used if a patient has a bleeding disorder, or when there is a need to measure the portal pressure gradient.

A third approach is having a surgeon perform a liver biopsy at the time of laparoscopy or laparotomy if the patient is already having surgery for another reason. For example, if a patient is undergoing a bariatric surgical procedure, there may be a desire to assess the liver for steatohepatitis.

The newest method is endoscopic ultrasound (EUS)guided liver biopsy. With this approach, an echoendoscope is used by an endosonographer to localize the liver. The needle can then be followed in real time as it obtains liver tissue.

G\&H Who is the ideal patient to undergo EUSguided liver biopsy? In whom is this method not appropriate?

DD The ideal patient to undergo EUS-guided liver biopsy is someone who is in need of both a liver biopsy and an endoscopic procedure. Examples include patients who require an upper endoscopy to investigate abdominal discomfort, to assess for chronic reflux, to rule out Barrett esophagus, to screen for esophageal varices, and to assess gastritis. Patients may require an EUS to evaluate the common bile duct due to elevated liver function tests. In any of these scenarios, it is much more convenient for the patient to undergo an esophagogastroduodenoscopy (EGD) or EUS to be followed immediately by EUS-guided liver biopsy. EUS-guided liver biopsy should be avoided in patients with a coagulopathy (platelets $<50,000$ or an international normalized ratio $>1.5$ ).

G\&H How is EUS-guided liver biopsy performed? 
DD A linear EUS endoscope is used to locate the left hepatic lobe from the proximal stomach or the right hepatic lobe through the duodenal bulb. It is essential to distinguish the left hepatic lobe from the spleen, as they both can be found from the proximal stomach, and the echotexture of the spleen and the liver can be very similar. If the spleen is misidentified as the liver, an inadvertent splenic puncture could occur, which could result in a bleeding complication. A 19-gauge EUS needle is selected to accomplish the parenchymal liver biopsy. The stylet is removed. Research has found that wet suction (priming the needle with saline or heparin) improves tissue yields, as does the use of a 19-gauge core biopsy needle. The vacuum syringe is set and attached to the needle with the stopcock closed. A suitable trajectory is found where the needle will travel during the biopsy, typically 3 to $5 \mathrm{~cm}$. Larger vessels are avoided when choosing this trajectory. The needle is then advanced through the gastric or duodenal wall, and just into the liver. The stopcock is turned on to deliver suction, and 1 to 3 to-andfro passes are made into liver tissue. Before removing the needle from the liver, the suction is turned off. The specimen is delivered straight into formalin from the 19-gauge needle without excessive handling.

\section{G\&H What are the advantages and disadvantages of EUS-guided liver biopsy compared to other methods?}

DD There are several advantages of the EUS-guided method. First, it is a sedated procedure, which may provide more comfort to the patient as well as being a less-invasive method of obtaining liver tissue. A sedated biopsy is also typically preferred for patients who are afraid of undergoing liver biopsy or who have trouble cooperating, such as in the pediatric population. A second advantage is that it uses real-time ultrasound guidance, allowing endoscopists to continuously follow the needle as it goes into the liver during the procedure, allowing identification of large vessels as well as other nearby organs. Third, 2 different areas of the liver can be sampled, which is harder to do with the percutaneous route, and is not possible with the transjugular route. Sampling 2 areas can decrease the potential of sampling error of liver biopsy. Fourth, EUS-guided liver biopsy is more convenient for patients who are undergoing an endoscopic procedure as well as a liver biopsy, as this method saves the need for 2 separate procedures. Finally, EUS-guided liver biopsy is performed within a gastrointestinal practice rather than being referred out.

The only disadvantage of EUS-guided liver biopsy at present is a slightly higher cost of the procedure in patients who do not need a concomitant EGD or EUS for another reason. The cost is due largely to the sedation of the patient, which can incur separate anesthesia charges.

G\&H What does the literature report regarding the safety of EUS-guided liver biopsy?

DD EUS-guided liver biopsy has been found to be extremely safe. In a multicenter study I conducted with my colleagues, there was only 1 episode of bleeding in a patient who had a coagulopathy. Only a handful of adverse effects have been described in the medical literature, and they are mainly bleeding events.

\section{G\&H What adverse events are associated with EUS-guided liver biopsy?}

DD Bleeding is the most common complication of liver biopsy by any route, although it appears to occur less frequently with EUS guidance than with the percutaneous route. Splenic puncture is an adverse event unique to the EUS method due to the possible misidentification of the spleen. However, this event can be completely avoided if the endoscopist double-checks that the correct organ is targeted. Percutaneous biopsy carries a risk of gallbladder puncture and pneumothorax that does not occur with EUS-guided liver biopsy due to the location of the involved organs and the route that is used with EUS.

G\&H How does the tissue yield of EUSguided liver biopsy compare to that of the percutaneous or transjugular method?

DD Multiple studies have evaluated EUS-guided liver biopsy and compared it to the other methods. My colleagues and I conducted a retrospective study comparing the adequacy of the specimen using the EUS-guided approach with that of the percutaneous and transjugular approaches. We found that the amount and quality of the liver tissue sampled with EUS guidance was at least the same, if not better, than that of the 2 other methods of liver biopsy.

\section{G\&H Does the size or type of the needle affect the diagnostic adequacy?}

DD Yes, it does. Most of the literature uses a 19-gauge needle. Tissue diameter delivered with this needle is comparable to that with an 18-gauge needle, which is used for most transjugular and percutaneous biopsies performed by radiology. Research has found that a 19-gauge core biopsy needle delivers better specimen in terms of intact specimen length. For an EUS-guided liver biopsy, the 19-gauge core biopsy needle is the preferred device. 
G\&H What is the learning curve associated with EUS-guided liver biopsy?

DD The learning curve varies depending on the EUSfine-needle aspiration experience and skill level of the endosonographer. In observing our advanced endoscopy fellows learning this technique, it appears that 12 to 15 procedures are needed to be able to identify landmarks, to properly actuate the needle in the liver, and to get reproducibly long specimen cores.

\section{G\&H Is EUS-guided liver biopsy used in clinical practice?}

DD My clinical practice uses EUS-guided liver biopsy extensively; nearly two-thirds of the liver biopsies performed in my institution are done by this route. The approach is also gaining traction at a lot of other centers, as more endoscopists are adopting EUS-guided liver biopsy.

\section{G\&H What are the priorities of research in this field?}

DD It would be beneficial to determine the ideal needle and the best method for sticking the liver and managing the specimen. Through a series of studies, we have figured out that a 19-gauge core needle is better than both a 19 -gauge regular needle and a 22 -gauge core needle, that wet suction is better than dry suction, and that 3 actuations of a needle is better than 1 actuation of a needle. More work is needed to figure out the correct amount of suction to apply to the needle when performing the biopsy as well as to confirm the number of actuations. There are several ways of accomplishing the biopsy with a different number of actuations of the needle and various methods of puncturing the liver, and they all seem to work. The other area of research that is needed is an analysis of the cost benefits of using EUS-guided liver biopsy plus endoscopy vs using those 2 procedures separately. I think that we would find a cost advantage in performing the EUS-guided liver biopsy in patients who need endoscopy or EUS. Another study that is worth conducting is a patient satisfaction study comparing the various routes of liver biopsy.

\section{G\&H What technology is on the horizon?}

DD There is an emerging technology for measuring the portal pressure gradient with EUS guidance. This involves taking pressure measurements from a hepatic vein and the portal vein with a 25 -gauge EUS needle. Further development of this method would add another dimension to liver assessment by EUS. Portal pressure measurement can currently only be conducted through the transjugular route. However, if it can be done all through a sedated endoscopy, during which time a liver biopsy is also taken, this would be an easier and much more comfortable method for the patient. The technology to accomplish this is in development, but I expect to see it available for clinical use within a year.

Dr Diehl serves as a consultant for Boston Scientific, Medtronic, Cook Medical, Olympus America, and Pentax.

\section{Suggested Reading}

Diehl DL, Johal AS, Khara HS, et al. Endoscopic ultrasound-guided liver biopsy: a multicenter experience. Endosc Int Open. 2015;3(3):E210-E215.

Johal A, Diehl D, Khara HS. EUS-guided liver biopsy. www.broadcastmed.com/ gastroenterology/6663/videos/eus-guided-liver-biopsy-by-amit-singh-johal-mddavid-diehl-md-harshit-s-khara-md. Published September 19, 2017. Accessed November 19, 2018

Johal AS, Khara HS, Maksimak MG, Diehl DL. Endoscopic ultrasound-guided liver biopsy in pediatric patients. Endosc Ultrasound. 2014;3(3):191-194.

Lee WJ, Uradomo LT, Zhang Y, Twaddell W, Darwin P. Comparison of the diagnostic yield of EUS needles for liver biopsy: ex vivo study. Diagn Ther Endosc. 2017;2017:1497831

Mohan BP, Shakhatreh M, Garg R, Ponnada S, Adler DG. Efficacy and safety of EUS-guided liver biopsy: a systematic review and meta-analysis [published online October 30, 2018]. Gastrointest Endosc. doi:10.1016/j.gie.2018.10.018.

Pineda JJ, Diehl DL, Miao CL, et al. EUS-guided liver biopsy provides diagnostic samples comparable with those via the percutaneous or transjugular route. Gastrointest Endosc. 2016;83(2):360-365. 